\title{
TABLEBASE OF CONTENTS of the ICCA Journal
}

\author{
Collected by Jos Uiterwijk \\ Deputy Editor
}

This collection contains the references to all (scientific) Articles, Notes and Literature Reviews, appeared in the ICCA Journal, starting from Vol. 6, No. 3 (August 1983) up to and including Vol. 12, No. 4 (December 1989).

The list is alphabetically ordered. The symbol following each reference stands for: Article (A), Note (N) or Review (R). The publications before Volume 9 are not distinguished into Articles and Notes; then a marking symbol has been omitted.

Althöfer, I. (1988a). Root Evaluation Errors: How they Arise and Propagate. ICCA Journal, Vol. 11, No. 2/3, pp. 55-63. (A)

Althöfer, I. (1988b). Günther Schrüfer: Minimax-suchen: Kosten, Qualität und Algorithmen. ICCA Journal, Vol. 11, No. 4, pp. 160-161 (R)

Althöfer, I. (1989). Retrograde Analysis and Two Computerizable Definitions of the Quality of Chess Games. ICCA Journal, Vol. 12, No. 2, pp. 74-78. (N)

Anantharaman, T.S., Campbell, M.S. and Hsu, F. (1988). Singular Extensions: Adding Selectivity to BruteForce Searching. ICCA Journal, Vol. 11, No. 4, pp. 135-143. (A)

Bal, H.E. and Renesse, R. van (1986). A Summary of Parallel Alpha-Beta Search Results. ICCA Journal, Vol. 9, No. 3, pp. 146-149. (A)

Beal, D.F. (1984a). Mixing Heuristic and Perfect Evaluations: Nested Minimax. ICCA Journal, Vol. 7, No. 1, pp. 10-15.

Beal, D.F. (1984b). Mating Sequences in the Quiescence Search. ICCA Journal, Vol. 7, No. 3, pp. 133-137.

Beal, D.F. (1986a). Selective Search without Tears. ICCA Journal, Vol 9, No. 2, pp. 76-80. (A)

Beal, D.F. (1986b). M.M. Botvinnik: Computers in Chess - solving inexact search problems. ICCA Journal, Vol. 9, No. 2, pp. 88-89. (R)

Bergen, A.R.D. van (1985). An Ultimate Look at the KPK Data Base. ICCA Journal, Vol. 8, No. 4, pp. 216 218.

Bergen, A.R.D. van and Storm, T. van der (1986). The KPK Endgame: a Unit Correction. ICCA Journal, Vol. 9, No. 1, pp. 35-36. (N)

Berliner, H. (1987). Some Innovations Introduced by Hitech. ICCA Journal, Vol. 10, No. 3, pp. 111-117. (A)

Bettadapur, P. (1986). Influence of Ordering on Capture Search. ICCA Journal, Vol. 9, No. 4, pp. 180-188. (A)

Botvinnik, M.M. (1983). The Game of Chess. Its past, present and future. ICCA Journal, Vol. 6, No. 3, pp. 69.

Bramer, M.A. (1986). KPK Endgame Databases: a response from the source. ICCA Journal, Vol. 9, No. 3, pp. 150-151. (N)

Bratko, I., Tancig, P. and Tancig, S. (1984). Detection of Positional Patterns in Chess. ICCA Journal, Vol. 7, No. 2, pp. 63-73.

Cracraft, S.M. (1984). Bitmap Move Generation in Chess. ICCA Journal, Vol. 7, No. 3, pp. 146-153.

Dekker, S.T., Herik, H.J. van den and Herschberg, I.S. (1987). Complexity Starts at Five. ICCA Journal, Vol. 10, No. 3, pp. 125-138. (A)

Donskoy, M. and Schaeffer, J. (1989). Perspectives on Falling from Grace. ICCA Journal, Vol. 12, No. 3, pp. 155-163. (N)

Falk, L. and The Editorial Board (1989). So Help Me the Database. ICCA Journal, Vol. 12, No. 3, pp. $165-$ 166. (N)

Feldmann, R., Monien, B., Mysliwietz, P. and Vornberger, O. (1989) Distributed Game-Tree Search. ICCA Journal, Vol. 12, No. 2, pp. 65-73. (A)

Forthoffer, D., Rasmussen, L. and Dekker, S.T. (1989). A Correction to some KRKB-Database Results. ICCA Journal, Vol. 12, No. 1, pp. 25-27. (N)

Frey, P.W. (1985). An Empirical Technique for Developing Evaluation Functions. Some thoughts with a review of Mitchell's thesis. ICCA Journal, Vol. 8, No. 1, pp. 17-22. 
Frey, P.W. (1986). Fuzzy Production Rules in Chess. ICCA Journal, Vol 9, No. 4, pp. 175-179. (A)

Good, I.J. (1988). Some Comments Concerning an Article by De Groot. ICCA Journal, Vol. 11, No. 2/3, p. 80. (N)

Grand, H. le (1986). The Impact of Computers on Chess-Problem Composition. ICCA Journal, Vol. 9, No. 3, pp. 152-153. (N)

Groot, A.D. de (1986). Intuition in Chess. ICCA Journal, Vol. 9, No. 2, pp. 67-75. (A)

Groot, A.D. de (1987). Some Benefits of Advances in Computer Chess. ICCA Journal, Vol. 10, No. 2, pp. 72-77. (A)

Groot, A.D. de (1988). A Rejoinder to I.J. Good's Comments. ICCA Journal, Vol. 11, No. 2/3, p. 81. (N)

Hartmann, D. (1987a). How to Extract Relevant Knowledge from Grandmaster Games. Part 1: Grandmasters have insights - the problem is what to incorporate into practical programs. ICCA Journal, Vol 10, No. 1, pp. 14-36. (A)

Hartmann, D. (1987b). D.F. Beal (Editor): Advances in Computer Chess 4. ICCA Journal, Vol. 10, No. 1, pp. 41-42. (R)

Hartmann, D. (1987c). How to Extract Relevant Knowledge from Grandmaster Games. Part 2: The notion of mobility, and the work of De Groot and Slater. ICCA Journal, Vol. 10, No. 2, pp. 78-90. (A)

Hartmann, D. (1987d). R. Bartel, H.J. Kraas and G. Schrüfer: Das grosse Computerschach-Buch. ICCA Journal, Vol. 10, No. 2, pp. 96-97. (R)

Hartmann, D. (1987e). Peter van Diepen and Jaap van den Herik: Schaken voor Computers. ICCA Journal, Vol. 10, No. 3, p. 143. (R)

Hartmann, D. (1987f). Reiner Seidel: Grundlagen einer wissenschaftlichen Schachtheorie. ICCA Journal, Vol. 10, No. 4, pp. 195-196. (R)

Hartmann, D. (1988a). Christian Posthoff and Günter Reinemann: Computerschach - Schachcomputer. ICCA Journal, Vol. 11, No. 1, pp 34-35. (R)

Hartmann, D. (1988b). Rejoinder to Seidel's Reply. ICCA Journal, Vol 11, No. 1, pp. 38-39. (R)

Hartmann, D. (1988c). Butterfly Boards. ICCA Journal, Vol. 11, No. 2/3, pp. 64-71. (A)

Hartmann, D. (1988d). Alen D. Shapiro: Structured Induction in Expert Systems. ICCA Journal, Vol. 11, No. 4, pp. 163-164. (R)

Hartmann, D. (1989a). David N.L. Levy (Editor): Computer Chess Compendium. ICCA Journal, Vol. 12, No. 1, pp. 27-28. (R)

Hartmann, D. (1989b). J.E. Hayes, D. Michie and J. Richards (Editors): Machine Intelligence 11: Logic and the Acquisition of Knowledge. ICCA Journal, Vol. 12, No. 3, pp. 168-169. (R)

Herik, H.J. van den (1986). Roycroft's 5-Man Chess Endgame Series. ICCA Journal, Vol. 9, No. 3, pp. 154155. (R)

Herik, H.J. van den and Herschberg, I.S. (1985a). The Construction of an Omniscient Endgame Data Base. ICCA Journal, Vol. 8, No. 2, pp 66-87.

Herik, H.J. van den and Herschberg, I.S. (1985b). Elementary Theory Improved, a Conjecture Refuted. ICCA Journal, Vol. 8, No. 3, pp 141-149.

Herik, H.J. van den and Herschberg, I.S. (1986a). A Data Base on Data Bases. ICCA Journal, Vol. 9, No. 1, pp. 29-34. (A)

Herik, H.J. van den and Herschberg, I.S. (1986b). A Note on KBBK. ICCA Journal, Vol. 9, No. 4, p. 199. (N)

Herik, H.J. van den and Herschberg, I.S. (1987). The KBBKN Statistics: New data from Ken Thompson. ICCA Journal, Vol. 10, No. 1, pp. 39-40. (N)

Herik, H.J. van den and Herschberg, I.S. (1988). Computer Checks on Human Analyses of the KRKB Endgame. ICCA Journal, Vol. 11, No. 1, pp. 26-31. (N)

Herik, H.J. van den, Herschberg, I.S. and Nakad, N. (1987). A Six-Men-Endgame Database: KRP(a2)KbBP(a3).ICCA Journal, Vol. 10, No. 4, pp. 163-180. (A)

Herik, H.J. van den, Herschberg, I.S. and Nakad, N. (1988a). Karpov Amends Timman's Analysis. ICCA Journal, Vol. 11, No. 1, pp. 32-33. (N)

Herik, H.J. van den, Herschberg, I.S. and Nakad, N. (1988b). A Reply to R. Sattler's Remarks on the $\operatorname{KRP}(\mathrm{a} 2) \mathrm{KbBP}(\mathrm{a} 3)$ Database. ICCA Journal, Vol. 11, No. 2/3, pp. 88-91. (N)

Herik, H.J. van den and Jong-Gierveld, J. de (1983). Computer Chess: Trick or Treat? Preliminary thoughts on threats and rewards. ICCA Journal, Vol. 6, No. 4, pp. 15-22.

Herik, H.J. van den and Jong-Gierveld, J. de (1984a). Computer Chess: Trick or Treat? Part II: threats and fears investigated. ICCA Journal, Vol. 7, No. 1, pp. 16-22. 
Herik, H.J. van den and Jong-Gierveld, J. de (1984b). Computer Chess: Trick or Treat? Part III: some fears revealed. ICCA Journal, Vol 7, No. 2, pp. 74-83.

Herschberg, I.S. (1986). The $5^{\text {th }}$ World Microcomputer Chess Championship. ICCA Journal, Vol. 9, No. 3, p. 155 . (R)

Herschberg, I.S. and Herik, H.J. van den (1985). A Gauge of Endgames. ICCA Journal, Vol. 8, No. 4, pp. 225-229.

Herschberg, I.S. and Herik, H.J. van den (1986). Thompson's New Data-Base Results. ICCA Journal, Vol. 9 , No. 1, pp. 45-49. (N)

Herschberg, I.S. and Herik, H.J. van den (1987). More Truth on KBBK Database Results. ICCA Journal, Vol. 10, No. 2, pp. 91-93. (N)

Herschberg, I.S., Herik, H.J. van den and Schoo, P.N.A. (1989). Verifying and Codifying Strategies in the KNNKP(h) Endgame. ICCA Journal, Vol. 12, No. 3, pp. 144-154. (A)

Horacek, H. (1983). Knowledge-Based Move Selection and Evaluation to Guide the Search in Chess Pawn Endings. ICCA Journal, Vol. 6, No 3, pp. 20-37.

Hünen, R.W.G. (1987). A Uniform Way to Report Database Results. ICCA Journal, Vol. 10, No. 2 , p. 91. (N)

Hyatt, R.M. (1984). Using Time Wisely. ICCA Journal, Vol. 7, No. 1, pp 4-9.

Hyatt, R.M. (1985). Parallel Chess on the Cray X-MP/48. ICCA Journal, Vol. 8, No. 2, pp. 90-99.

Kaindl, H. (1988). Useful Statistics from Tournament Programs. ICCA Journal, Vol. 11, No. 4, pp. 156-159. (N)

Kaindl, H., Horacek, H. and Wagner, M. (1986). Selective Search versus Brute Force. ICCA Journal, Vol. 9 , No. 3, pp. 140-145. (A)

Komissarchik, E.A. and Futer, A.L. (1986). Computer Analysis of a Queen Endgame. ICCA Journal, Vol. 9, No. 4, pp. 189-198. (A)

Larsson, J.E. (1987). Challenging that Mobility is Fundamental. ICCA Journal, Vol. 10, No. 3, pp. 139-142. (N)

Levinson, R.A. (1989). A Self-Learning, Pattern-Oriented Chess Program. ICCA Journal, Vol. 12, No. 4, pp. 207-215. (A)

Levy, D.N.L. (1986). When Will Brute Force Programs Beat Kasparov? ICCA Journal, Vol. 9, No. 2, pp. 8186. (A)

Levy, D.N.L. (1987). Improving the Performance of Endgame Databases. ICCA Journal, Vol. 10, No. 4, pp. 191-192. (N)

Levy, D.N.L., Broughton, D. and Taylor, M. (1989). The SEX Algorithm in Computer Chess. ICCA Journal, Vol. 12, No. 1, pp. 10-21. (A)

Lindner, L. (1983). Experience with the Second Human-Computer Problem Test. ICCA Journal, Vol. 6, No. 3, pp. 10-15.

Lindner, L. (1985). A Test to Compare Human and Computer Fairy-Chess Problem Solving. ICCA Journal, Vol. 8, No. 3, pp. 150-155.

Marris, Ch.A. (1989). Compressing a Chess-Endgame Database. ICCA Journal, Vol. 12, No. 1, pp. $22-24$. (N)

Marsland, T.A. (1983). P.W. Frey (Ed.): Chess Skill in Man and Machine (2nd ed.). ICCA Journal, Vol. 6, No. 4, pp. 46-48. (R)

Marsland, T.A. (1984a). David E. Welsh: Computer Chess. ICCA Journal, Vol. 7, No. 1, pp. 36-37. (R)

Marsland, T.A. (1984b). David Levy: The Chess Computer Handbook. ICCA Journal, Vol. 7, No. 2, p. 84. (R)

Marsland, T.A. (1984c). David Levy: Computer Gamesmanship. ICCA Journal, Vol. 7, No. 2, pp. 84-85. (R)

Marsland, T.A. (1984d). M.A. Bramer (Editor): Computer Game-Playing: Theory and Practice. ICCA Journal, Vol. 7, No. 2, p. 85. (R)

Marsland, T.A. (1984e). H. Berliner and M. Campbell: Using Chunking to Solve Chess Pawn Endings. ICCA Journal, Vol. 7, No. 3, p. 154. (R)

Marsland, T.A. (1985a). R.M. Hyatt: Cray Blitz - A Computer Chess Playing Program. ICCA Journal, Vol. 8, No. 1, pp. 23-24. (R)

Marsland, T.A. (1985b). Evaluation-Function Factors. ICCA Journal, Vol 8, No. 2, pp. 47-57.

Marsland, T.A. (1985c). D.E. Welsh and B. Baczynskyj: Computer Chess II. ICCA Journal, Vol. 8, No. 3, p. 156. (R)

Marsland, T.A. (1986). A Review of Game-Tree Pruning. ICCA Journal, Vol. 9, No. 1, pp. 3-19. (A) 
Marsland, T.A. (1987a). L. Pachman and V. Kuhnmund: Computer Chess. ICCA Journal, Vol. 10, No. 2, p. 97. (R)

Marsland, T.A. (1987b). Carl Ebeling: All the Right Moves: A VLSI Architecture for Chess. ICCA Journal, Vol. 10, No. 4, p. 197. (R)

Marsland, T.A. (1989a). D.F. Beal (Editor): Advances in Computer Chess 5. ICCA Journal, Vol. 12, No. 3, p. 169. (R)

Marsland, T.A. (1989b). Levy (Editor): Computer Chess Compendium. ICCA Journal, Vol. 12, No. 4, p. 218. (R)

McCarthy, J. (1989). The Fruitfly on the Fly. ICCA Journal, Vol. 12, No. 4, pp. 199-206. (A)

Michie, D. (1989). Brute Force in Chess and Science. ICCA Journal, Vol 12, No. 3, pp. 127-143. (A)

Michie, D. and Bratko, I. (1987a). Ideas on Knowledge Synthesis Stemming from the KBBKN Endgame. ICCA Journal, Vol. 10, No. 1, pp 3-13. (A)

Michie, D. and Bratko, I. (1987b). Ideas on Knowledge Synthesis ..... a correction. ICCA Journal, Vol. 10, No. 2, p. 94. (N)

Nefkens, H.J.J. (1985). Constructing Data Bases to Fit a Microcomputer. ICCA Journal, Vol. 8, No. 4, pp. 219-224.

Nelson, H.L. (1985). Hash Tables in Cray Blitz. ICCA Journal, Vol. 8, No. 1, pp. 3-13.

Nelson, H.L. and Hyatt, R.M. (1988). The Draw Heuristic of Cray-Blitz. ICCA Journal, Vol. 11, No. 1, pp. 3-9. (A)

Newborn, M.M. (1985). A Hypothesis Concerning the Strength of Chess Programs. ICCA Journal, Vol. 8, No. 4, pp. 209-215.

Posthoff, C. (1988a). G.M. Adelson-Velsky, V.L. Arlazarov and M.V Donskoy: Algorithms for Games. ICCA Journal, Vol. 11, No. 2/3, pp 92-93. (R)

Posthoff, C. (1988b). David N.L. Levy (Editor): Computer Games I. ICCA Journal, Vol. 11, No. 2/3, pp. $94-$ 95. (R)

Rasmussen, L. (1987). Correcting Grandmasters' Analyses in Elementary Endgames. ICCA Journal, Vol. 10, No. 4, pp. 192-194. (N)

Rasmussen, L. (1988a). Ultimates in KQKR and KRKN. ICCA Journal, Vol 11, No. 1, pp. 21-25. (N)

Rasmussen, L. (1988b). A Database for KRKP. ICCA Journal, Vol. 11, No 4, pp. 144-150. (A)

Reed, A. (1986). A Response to the Bergen/Storm KPK Endgame Result. ICCA Journal, Vol. 9, No. 2, p. 87. (N)

Reinefeld, A. (1983). An Improvement to the Scout Tree-Search Algorithm. ICCA Journal, Vol. 6, No. 4, pp. 4-14.

Reinefeld, A. (1985). Kai von Luck: Aspekte wissensgestutzter Planung. Bernd Owsnicki: Repräsentation von positionellem Schachwissen mit Techniken der kunstlichen Intelligenz. ICCA Journal, Vol. 8, No 4, pp. 235-236. (R)

Roycroft, A.J. (1987). The Expcrimental Positions of Ideas in Knowledge Synthesis. ICCA Journal, Vol. 10, No. 2, p. 95. (N)

Sattler, R. (1988). Further to the KRP(a2)KbBP(a3) Database. ICCA Journal, Vol. 11, No. 2/3, pp. 82-87. (N)

Schaeffer, J. (1983). The History Heuristic. ICCA Journal, Vol. 6, No 3, pp. 16-19.

Schaeffer, J. (1984). The Relative Importance of Knowledge. ICCA Journal, Vol. 7, No. 3, pp. 138-145.

Schaeffer, J. (1985). Lionel Moser: An Experiment in Distributed Game Tree Searching. ICCA Journal, Vol. 8, No. 2, pp. 88-89. (R)

Schaeffer, J. (1987). Speculative Computing. ICCA Journal, Vol. 10, No 3, pp. 118-124. (A)

Schaeffer, J. (1988). G.M. Adelson-Velsky, V.L. Arlazarov and M.V Donskoy: Algorithms for Games. ICCA Journal, Vol. 11, No. 2/3, pp 93-94. (R)

Schaeffer, J. (1989). Comment on 'Distributed Game-Tree Search'. ICCA Journal, Vol. 12, No. 4, pp. 216217. (N)

Schlosser, M. (1988). Computers and Chess-Problem Composition. ICCA Journal, Vol. 11, No. 4, pp. 151155. (N)

Schrüfer, G. (1989). A Strategic Quiescence Search. ICCA Journal, Vol 12, No. 1, pp. 3-9. (A)

Seidel, R. (1985a). Chess, How to Understand the Exceptions! ICCA Journal, Vol. 8, No. 1, pp. 14-16.

Seidel, R. (1985b). Grammatical Description of Chess Positions. Database versus human knowledge. ICCA Journal, Vol. 8, No. 3, pp. 127-140.

Seidel, R. (1986). What Constitutes Optimal Play? ICCA Journal, Vol. 9, No. 1, pp. 37-44. (N)

Seidel, R. (1988). Reply to D. Hartmann's Review of "Grundlagen einer wissenschaftlichen Schachtheorie". ICCA Journal, Vol. 11, No. 1, pp. 36-37. (R) 
Skiena, S.S. (1986). An Overview of Machine Learning in Computer Chess. ICCA Journal, Vol. 9, No. 1, pp. 20-28. (A)

Slate, D.J. (1984). Interior-Node Score Bounds in a Brute-Force Chess Program. ICCA Journal, Vol. 7, No. 4, pp. 184-192.

Slate, D.J. (1987). A Chess Program that Uses its Transposition Table to Learn from Experience. ICCA Journal, Vol. 10, No. 2, pp. 59-71. (A)

Stiller, L. (1989). Parallel Analysis of Certain Endgames. ICCA Journal, Vol. 12, No. 2, pp. 55-64. (A)

Szabo, A. and Szabo, B. (1988). The Technology Curve Revisited. ICCA Journal, Vol. 11, No. 1, pp. 14-20. (A)

The Editors (1987). Given a Queen, what Price the Issue? ICCA Journal, Vol. 10, No. 2, p. 95. (N)

Thompson, K. (1986a). Retrograde Analysis of Certain Endgames. ICCA Journal, Vol. 9, No. 3, pp. 131139. (A)

Thompson, K. (1986b). An Example of QPvQ. ICCA Journal, Vol. 9, No. 4, pp. 201-204. (N)

Uiterwijk, J.W.H.M. (1988). David N.L. Levy (Editor): Computer Games II. ICCA Journal, Vol. 11, No. 4, pp. 161-162. (R)

Uiterwijk, J.W.H.M. (1989). Hermann Kaindl: Problemlösen durch heuristische Suche in der artificial Intelligence. ICCA Journal, Vol 12, No. 3, pp. 170-171. (R)

Verhoef, T.F. and Wesselius, J.H. (1987). Two-Ply KRKN: Safely Overtaking Quinlan. ICCA Journal, Vol. 10, No. 4, pp. 181-190. (A)

Walker, A.N. (1984). Uniqueness in Game Trees. ICCA Journal, Vol. 7, No. 4, pp. 193-202.

Warnock, T. and Wendroff, B. (1988). Search Tables in Computer Chess. ICCA Journal, Vol. 11, No. 1, pp. 10-13. (A)

Wendroff, B. (1985). Attack Detection and Move Generation on the X-MP/48. ICCA Journal, Vol. 8, No. 2, pp. 58-65.

White, J.F. (1988). Querg Chess. ICCA Journal, Vol. 11, No. 2/3, pp 72-80. (A)

Wiereyn, P.H. (1985). Inventive Problem Solving. ICCA Journal, Vol. 8, No. 4, pp. 230-234.

Zellner, H. (1986). Compressing Databases down to Micro Size. ICCA Journal, Vol. 9, No. 4, pp. 199-200. (N)

Zellner, H. (1987). KBBK Squeezed into a Micro. ICCA Journal, Vol. 10, No. 1, pp. 37-39. (N)

Zellner, H. (1989a). The KPK Database Revisited. ICCA Journal, Vol. 12, No. 2, pp. 78-82. (N)

Zellner, H. (1989b). KBBKN on a Micro. ICCA Journal, Vol. 12, No. 3, pp. 163-164. (N)

Zellner, H., Herik, H.J. van den and Herschberg, I.S. (1987). Corrections and Substantiations to KBNK. ICCA Journal, Vol. 10, No. 3, p. 139. (N)

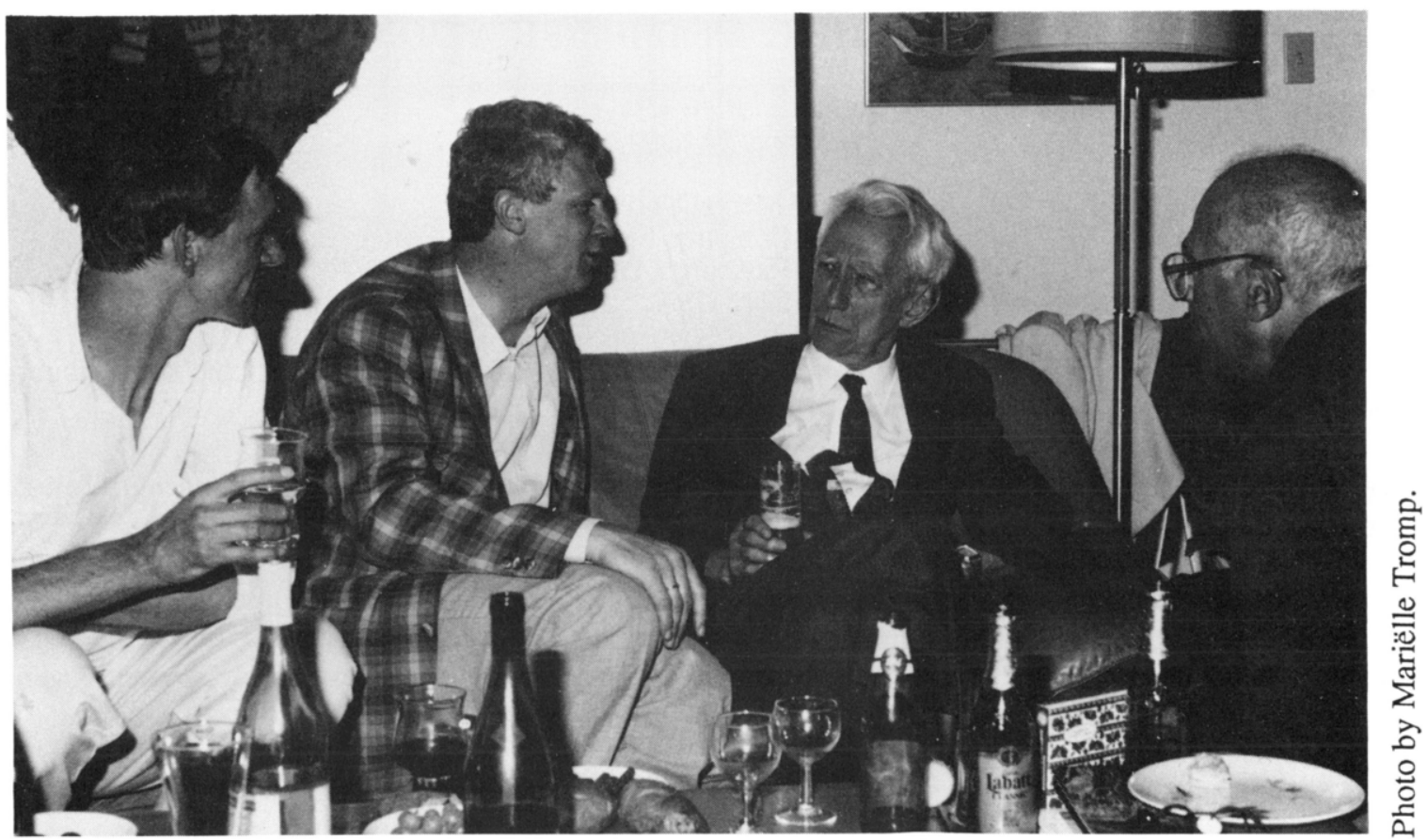

TALKS ON CHESS AND JUGGLING MIXED WITH BEER AND WINE.

A get-to-gether at Tony Marsland's house, Edmonton, Alberta, 1989.

Left to right: Jos Uiterwijk, Jaap van den Herik, Claude Shannon and Hans Berliner. 